\title{
Que la fuerza esté contigo: desvelar el lado oscuro de la investigación en educación
}

\section{Resumen}

En el presente artículo hacemos un recorrido en torno a la investigación cualitativa en Educación y planteamos cómo se enfrenta a una realidad caracterizada por políticas neoliberales que sumen a la universidad en la cultura de las auditorías. Estas condiciones de trabajo suponen para los investigadores - más en el caso de los noveles - presiones del sistema y de interpretación de su propia labor que conducen a dejarse llevar por el régimen establecido o a despertar el espíritu crítico y tratar de cambiarlo.

\section{Palabras clave descriptor}

Investigación, auditoría de gestión, universidad, investigación participativa.

\section{Transferencia a la práctica}

Los ideales económicos neoliberales que imperan en la actualidad se mueven en torno al mercado y a la competitividad. Esta situación dibuja un panorama para el investigador al servicio de intereses mercantilistas en términos de productividad científica. Para la investigación cualitativa, este discurso dominante es peligroso, ya que la mayoría de los estándares están preparados para otras formas de investigación. Es posible distinguir diferentes aspectos para reflexionar sobre la orientación de la investigación, puesto que es necesario elegir entre el acomodo a las presiones del sistema y la participación - la lucha por el afianzamiento de procesos más justos. Las investigaciones críticas apuntan hacia esta última dirección.

Para citar este artículo / To cite this article / Pour citer cet article / Para citar este artigo

Barba, J. J., González-Calvo, G., \& Barba-Martín, R. A. (2014). Que la fuerza esté contigo: desvelar el lado oscuro de la investigación en educación. magis, Revista Internacional de Investigación en Educación, 7(14), 125-140. 
Key words plus

Research, Management Audit, University, Participative Research.

\section{Abstract}

In this article we discuss about qualitative research in education by asking ourselves how to deal with a reality characterized by neoliberal policies that are added to the university culture of audits. These working conditions pose for researchers, more in the case of the beginners, system pressures and interpretation of their own work that lead them to be swayed by the established regime or, on the contrary, to awake their critical spirit to try to change it.

\section{Transference to practice}

Neoliberal economic ideals that prevail today are moving around the market and competitiveness. This situation offers a panorama where the researcher is at the service of mercantilist interests in terms of scientific productivity. To the field of qualitative research this dominant discourse is more dangerous, since most of the standards are prepared for other types of research. It is possible to distinguish different aspects to reflect on the orientation of the research, having to choose between the adaptation to the system pressures or, on the contrary, to participate in the consolidation of more just processes. Critical investigations point towards the latter direction.

\section{Mots clés descripteur}

Recherche, audits de gestion, université, recherche participative.

\section{Résumé}

Dans le présent article on fait un parcoure tout autour de la recherche qualitative en éducation montrant comment on fait face à une réalité caractérisée par les politiques néolibérales qui soumissent à l'université dans la culture des audits. Ces conditions de travail supposent pour les chercheurs -et encore plus dans le cas des débutantsdes contraints du système et d'interprétation de leur propre rôle qui les conduisent à se laisser aller par le régime établit, ou bien à se réveiller et avoir un esprit critique qui essaye de changer ce système.

\section{Transfert à la pratique}

Les enjeux économiques néolibéraux qui dominent dans l'actualité bougent tout autour du marché et la compétitivité. Cette situation dessine un panorama de recherche au service des intérêts du marché en termes de productivité scientifique. Pour la recherche qualitative ce discours dominant est plus dangereux, puisque la plupart des standards sont faits pour autres types de recherche. II est possible de distinguer les différents aspects et réfléchir sur la direction de la recherche, et il faut choisir entre la position d'être à l'aise avec les contraintes du système ou bien choisir la participation et la lutte pour consolider des processus plus justes. Les recherches visent cette dernière direction.

\section{Palavras-chave descritor} Pesquisa, auditoria de gerenciamento, universidade, pesquisa participativa.

\section{Resumo}

No presente artigo fazemos um percurso ao redor da pesquisa qualitativa em educação propondo como enfrentar uma realidade caracterizada por políticas neoliberais que ponha a universidade na cultura das auditorias. Estas condições de trabalho supõem para os pesquisadores - mais ainda no caso dos novatos - pressões do sistema e de interpretação de sua própria atividade, o que os conduzem a se deixar levar pelo regime estabelecido ou a despertar o espírito crítico e tratar de mudar.

\section{Transferência à prática}

Os ideais econômicos neoliberais que imperam na atualidade se movem em torno do mercado e da competitividade. Esta situação desenha um panorama de pesquisa a serviço de interesses mercantilistas em termos de produtividade científica. Para a pesquisa qualitativa este discurso dominante é mais perigoso, visto que os padrões a seguir estão preparados para outras formas de pesquisa. É possível distinguir diferentes aspectos para refletir sobre a orientação da pesquisa, devendo-se escolher entre a acomodação às pressões do sistema ou a participação e a luta pela consolidação de processos mais justos. As pesquisas críticas apontam para esta última direção. 


\section{Introducción}

La pedagogía crítica se resiste a la creciente mercantilización y comercialización de la educación superior. Lucha contra la penetración de los valores neoliberales en las zonas de investigación, en las aulas y en los currículos. Es crítica con los comités de evaluación institucional, cuyos juicios sobre investigaciones de las disciplinas humanísticas son todavía más restrictivos (Denzin, 2008, p. 196).

Si las personas vinculadas al ámbito de la Educación nos detuviéramos por un momento a preguntarnos qué es lo fundamental para enseñar e investigar dentro de nuestro campo, los diferentes profesionales tendríamos opiniones dispares acerca de los propósitos que buscamos. Se podría considerar que no hay una visión invariable de lo que ha de ser la enseñanza y, en consecuencia, la investigación educativa, pues esta lleva implícita una creencia particular de la sociedad, del ser humano y de la enseñanza, lo que conduce a diversas perspectivas sobre cómo ha de ser abordada. Estas formas distintas de entender los procesos educativos conllevan tres paradigmas de investigación identificados en la literatura (Latorre, Del Rincón \& Arnal, 1996), que son: positivista, interpretativo y sociocrítico. Es tradicional que la investigación cualitativa se adscriba al paradigma interpretativo o al sociocrítico. Este artículo tiene como fin exponer algunas características de estos dos paradigmas, así como las dificultades que experimentan los investigadores cuando llevan a cabo investigación cualitativa. No podemos perder de vista que, a medida que la investigación cualitativa avanza y se aplica en campos concretos, está generando nuevos paradigmas dentro de la investigación cualitativa (Denzin \& Lincoln, 2011).

La investigación cualitativa tiene planteamientos epistemológicos y ontológicos específicos y que se diferencian de otras formas de investigación. Podemos decir que se trata de una propuesta para comprender o transformar los acontecimientos, las normas y los contextos desde la propia perspectiva de los participantes. El principal objetivo de la investigación cualitativa es proporcionar observaciones y descripciones claras, detalladas y pormenorizadas de un contexto social en particular. Si pretendiéramos ir más allá y buscásemos un denominador común de todas las estrategias metodológicas, este sería que se trabaja con datos descriptivos (Taylor \& Bogdan, 1986) particulares del contexto (Flick, 2004). Tras aceptar estas dos características comunes, es posible diferenciar dos tendencias: una investigación que busca comprender una realidad sin modificarla (interpretativa) y una que se centra en cambiar, transformar la realidad, en la que el investigador participa activamente de los cambios junto con otros actores educativos (investigación participativa o transformativa). En el primer caso - el de la investigación interpretativa-, los investigadores suelen ser actores externos a la realidad estudiada y recogen en detalle las perspectivas o significaciones de los participantes de una realidad. Esta comprensión minuciosa puede constituirse en el primer paso, como considera Denzin (2010a), para luchar por un mundo más justo en términos sociales; sin embargo, esto no siempre sucede. Una de las principales dificultades radica en que los informes se redactan en formatos muy específicos, sobre todo como artículos de revistas científicas, que son propios del lenguaje de la comunidad científica, pero que excluyen a otras poblaciones (Kaltmeier, 2012). Desde su experiencia en diferentes sistemas educativos, Hargreaves y Shirley (2012) consideran que el cambio real en las escuelas proviene de la transferencia lateral de las prácticas de éxito de las escuelas cercanas y no de las publicaciones de impacto derivadas de las investigaciones.
Descripción del artículo | Article description | Description de l'article / Artigo descrição

En este artículo de investigación, los autores hacen un recorrido por la investigación cualitativa y analizan su realidad en el mundo de la Educación. Se deriva de la investigación "Las competencias docentes en la formación inicial del profesorado en Educación Física". 
La investigación interpretativa nos hace preguntarnos cuál es la transferencia real de las investigaciones educativas si no consiguen mejorar los centros educativos en donde se realizan.

En el segundo caso -el de la investigación participativa o transformativa-, los investigadores encuentran su sentido más profundo en transformar la realidad mientras investigan. No buscan conocer ni describir la realidad, sino que el propio proceso de investigación consiste en mejorar la realidad educativa desde dos perspectivas: 1) emancipatoria, si el investigador no se incluye en el grupo, y 2) transformativa, si investigador y participantes se consideran en la misma realidad (Mertens, Sullivan \& Stace, 2011). Paulo Freire (2007) afirmaba que la clave en este tipo de investigación es transformar la realidad, no obtener datos para narrar lo que sucede. La investigación es el propio proceso de transformación de un grupo concreto. En Educación, esta perspectiva se ha seguido desde: a) la investigación acción (Barba \& González-Calvo, 2013; Contreras-Domingo, 1994; Elliot, 1990; McKernan, 1999; Stringer, 2007), en la que expertos colaboran con docentes para mejorar las prácticas, y b) la investigación de los propios docentes sobre sus prácticas (González-Calvo \& Barba, 2014; Kincheloe, 2012; Schön, 1998; Stenhouse, 1987; Zeichner, 1993).

\section{Investigación cualitativa en educación y contexto neoliberal}

Somos conocedores de que ninguna metodología de investigación va a determinar la verdad absoluta respecto al carácter de las relaciones sociales y la interpretación del universo social. La investigación cualitativa, centrada en conocer las palabras y significaciones de los actores educativos, así como sus relaciones en el contexto en el que tienen lugar, lleva implícitas unas relaciones entre el investigador y lo investigado que le son propias y que dan origen a un tipo de interacción que no se presenta en otras formas de investigación (Wiesenfeld, 2000). El investigador cualitativo, además de investigar, forma parte de la realidad estudiada. Ahora bien, en el proceso de intentar comprender la perspectiva del otro, algunos investigadores cualitativos permanecen largo tiempo en el campo (por ejemplo, en la investigación etnográfica); esto permite entablar relaciones de cercanía con los informantes y que, en algunos casos, se comporten como un nativo más.

En cualquier caso, el investigador habrá de buscar la mayor objetividad posible en su estudio. Por este motivo, Goetz y LeCompte (1988) recomiendan dejar que pase un tiempo entre el trabajo de campo y la escritura del informe. Por su parte, Kusher (2009) considera difícil llegar a la objetividad, pues solo se puede aproximar a ella en la medida en que "se realiza un acto de subjetividad suprema" (p. 11), si bien tampoco es algo que pretendan todos los autores (Barba, 2013). Lo que sí parece un acuerdo común es la imposibilidad de separar al investigador de las influencias del contexto y de sus creencias.

A la hora de llevar a cabo investigación cualitativa en Educación, este contexto se caracteriza por políticas neoliberales que han impregnado no solo a los centros educativos y sus prácticas, sino también a las formas de investigar en torno a estos. La búsqueda de estándares de investigación y de indicadores objetivos está causando problemas de financiación de la investigación cualitativa, porque se separa del mito de la investigación generado por la perspectiva cuantitativa (Denzin, 2008; 2009). Así, en las políticas públicas en educación y los lineamientos de financiación es común encontrar la priorización de investigaciones que producen "datos duros" (cuantitativos) que puedan estandarizarse y aplicarse a una población que se considera homogénea y que no reconoce la diversidad o la complejidad 
de los contextos. Es, además, una investigación que no siempre es crítica, pues no cuestiona el statu quo y tampoco interviene para hacer una transformación de la realidad educativa.

Por su parte, el papel del investigador cualitativo se asienta en la crítica como una característica inherente del intelectual y, por ende, del investigador. Chomsky (2007) o Barnett y Parry (2014) consideran que el auténtico intelectual es el que toma una actitud crítica contra el poder hegemónico al expresar sus propias inquietudes; de no ser así, el intelectual se convierte en un comisario cultural cuyos trabajos se encuentran al servicio del poder. Consideramos conveniente recordar la obra de Freire (2007) en la que explica que la neutralidad en las relaciones sociales es una forma implícita de ponerse de parte del opresor. Ante estos argumentos, se entiende que el papel del investigador no solo se basa en la producción de conocimiento científico, sino que se halla en un cruce de caminos en el que ha de optar por estar al servicio de los poderes hegemónicos o ser un elemento de transformación social. No queda más opción que la elección entre una de las dos vías, puesto que la neutralidad en las Ciencias Sociales es un imposible (Chomsky, 2007).

El dilema entre la reproducción o la justicia social como producto asociado con el resultado final de la investigación está presente en muchas de las decisiones que ha de tomar el investigador, pero suelen omitirse por el peso de la tradición o las presiones del sistema. Creemos importante recurrir al concepto de habitus de Bourdieu (2006), asumido como el conjunto de hábitos y creencias no explícitas que determinan la forma de entender una profesión. Sobre este particular, nos parece que se puede aprender mucho de acompañar a un estudiante de doctorado en su proceso de formación como investigador. Resulta muy interesante comprobar cómo la novedad y los cuestionamientos iniciales en cuanto a los roles del investigador se admiten por supuestos y se adaptan al funcionamiento tradicional, aunque este no se encuentre en ningún código escrito y se aproxime al funcionamiento del resto del colectivo. Este proceso de inserción en el grupo investigador es sutil y transmite unos valores que, poco a poco, son aceptados de forma acrítica. Por ejemplo, es común que un estudiante de maestría se plantee cuestiones éticas relacionadas con la investigación; sin embargo, tiempo después casi nadie se cuestiona el anonimato en la investigación, cuando este es incompatible con alguna forma de investigación cualitativa como la autoetnografía.

En las próximas páginas estableceremos un diálogo con el lector entre la situación actual de la investigación cualitativa y las posibles formas de afrontarla y propondremos una serie de aspectos que, en muchas ocasiones, se mantiene oculta en los procesos de investigación y se dan por sentados, pero que han de ser fruto de una profunda reflexión en los próximos años, en busca del posicionamiento de los investigadores.

\section{La cara oculta de la investigación cualitativa}

En esta sección abordaremos algunos desafíos para el investigador cualitativo en Educación en torno a dos ejes: las presiones del sistema y la figura del investigador.

\section{Las presiones del sistema}

El sistema en el que se encuentra el investigador condiciona muchas de sus decisiones y formas de actuar. A continuación exponemos los cuatro aspectos más influyentes: 
- Investigar para dominar el mundo. Hasta este punto hemos considerado la relación entre la investigación cualitativa con la comprensión profunda de la realidad como un primer paso para transformar la realidad en una más justa. No obstante, también puede ser utilizada para la dominación de grupos sociales mediante el conocimiento profundo que se obtiene (Denzin \& Lincoln, 2011; Villasante \& Montañés, 2000) o del lenguaje que se utiliza para narrar la investigación (Potter, 1996). Podemos pensar en un publicista que organiza grupos de discusión con diferentes sectores sobre un producto que quiere vender. El inmenso conocimiento que obtiene sobre las relaciones de las personas con el producto no tiene como fin fomentar la justicia social, sino conocer cuáles son los valores que representa, qué imagen social crea, cuáles aspectos son percibidos como positivos por las personas, etc. De ese conocimiento presentará una campaña publicitaria que trate de hacernos consumir, mas no fomentará la igualdad, la equidad, la emancipación, etc.

En Educación sucede algo parecido cuando algunas compañías de tecnología patrocinan congresos, jornadas o cursos sobre alguno de sus productos, con el fin de presentar investigaciones que fomenten las virtudes que tienen y ser un escaparate de apoyo de la investigación. En esos congresos solo se presenta una visión del producto, que suele ser muy favorable para el patrocinador.

- La universidad becaria. En la famosa serie de dibujos animados, el coyote nunca consiguió atrapar al correcaminos, aun cuando estaba sumido en una dinámica de probar todos los productos que fabricaban en ACME. No obstante, la caza del correcaminos hubiera sido fácil si la situación se hubiera reflexionado y estudiado con calma. De ser así, habría sabido que el coyote puede correr a $69 \mathrm{~km} / \mathrm{h}$ y el correcaminos a $32 \mathrm{~km} / \mathrm{h}$, por lo que, con confianza en sus posibilidades y esta información, no habría necesitado artefactos.

Este relato de una serie de dibujos animados sirve como metáfora para plantearnos que las empresas buscan un beneficio económico de las investigaciones y no una mayor justicia social, aunque ella pueda enmascararlo. La relación entre empresa y universidad considera a esta última como una sucursal de investigación de bajo coste a su servicio y sin capacidad crítica. Sirva de ejemplo una situación vivida hace poco en un acto académico: uno de nosotros presentó un alegato en contra de los recortes en educación por la situación económica en España y la respuesta obtenida fue la expulsión, por un grupo de representantes de una entidad financiera, que había cedido el edificio a la universidad. En este caso se acusó de falta de respeto, pero lo que subyacía era poner un bozal a una institución que tiene un papel clave en la formación del pensamiento crítico (Greenwood, 2012).

La relación entre universidad y empresa, entendida como la primera al servicio de la segunda, está generando una transformación en la que las competencias relacionadas con el mercado laboral se afianzan, mientras que las que promueven una sociedad más justa quedan obsoletas y se les omite (Barnett, 2001). En la investigación, Grennwood (2012) afirma que la prioridad de esta se encuentra en aquello que puede producir resultados rápidos e ingresos económicos. De este modo, al solicitar proyectos de investigación o en los currículos de los investigadores en España se valoran las patentes generadas y la formación de spin-off, esto es, empresas derivadas de la explotación de patentes. Al reflexionar sobre esta línea de pensamiento nos preguntamos cuál es el papel de la investigación crítica para transformar los contextos sociales y las relaciones entre participantes, 
si esta no puede medirse en términos económicos. En el caso de la Educación, medir resultados en términos económicos es complejo, ya que desde que se implementan medidas en una escuela infantil, hasta que se comprueba su eficacia por la incorporación de esos estudiantes al mercado laboral, pasa más de una década.

Otra forma de relación universidad-empresa se observa en la producción de bienes y servicios y la obtención de beneficios económicos. Esto se efectúa por medio de mercados de productos que pueden desarrollarse y ser vendidos a corto plazo con la posibilidad de ser estudios patrocinados por empresas. Este es el trasfondo de los libros de texto y el de programas y soportes informáticos. Ya en la década del ochenta Apple (1989) nos alertaba sobre los libros de texto y su papel en imponer una cultura hegemónica de grupos poderosos. Resulta peligrosa la relación entre empresa y universidad, pues el mercado de productos educativos conlleva la asunción de valores y formas de pensamiento de los grupos hegemónicos. Como investigadores, hemos de tener en cuenta cuál es nuestra relación con las empresas, porque al asumirlas como propias de modo pasivo y de manera acrítica, "el valor mercantil de las investigaciones prevalece sobre su contenido de verdad" (Díez-Gutiérrez, 2011, p. 70).

- Publicar o morir. Hemos comenzado este párrafo con lo que Lawrence (2008) considera la regla que se está imponiendo en la actualidad. Cuando la investigación se mide en términos de publicaciones en revistas de impacto y no de otra serie de criterios, el investigador puede cambiar la consecuencia por la causa. Mientras las publicaciones han resultado de investigaciones realizadas, hoy día, con las reglas impuestas, pueden convertirse en el objetivo de investigar. No obstante, en la actualidad se presenta la característica de que las propias revistas están marcando qué deben hacer los investigadores si quieren publicar. Empieza a ser habitual comprobar en las normas de publicación que el autor debe conocer las publicaciones de la revista, lo que lleva implícita la citación de artículos para elevar índices de impacto. Este aspecto puede ser interesante, pero no cuando se utiliza como un criterio para rechazar artículos en una revisión por el editor. En varias ocasiones nos hemos encontrado con el rechazo de manuscritos con el argumento de que no conocíamos los artículos de la revista. De forma sutil nos indicaban que en esas revistas no publicaban artículos cualitativos, pese a que en sus normas de publicación no lo explicitaban. Esto puede suponer que las revistas no solo restringen la publicación en función de su temática en el campo de la Educación, sino que proponen metodologías y formas de escribir las investigaciones. Esta crítica la plantea Denzin (2010b), al considerar que las revisiones ciegas de artículos deben ser libres para que la valoración del trabajo se haga en función del manuscrito y no de los intereses de la revista.

La necesidad de publicar puede transformar por completo la forma de investigar, al desarrollar proyectos que puedan producir resultados de forma rápida (Greenwood, 2012) o que sean parcializados en forma de artículos (Lawrence, 2008). Esto último puede promover situaciones del tipo: a) los investigadores pueden ser clasificados por el índice de impacto que tienen sus obras, como afirma Sparkes (2013) respecto a la extensión del uso del índice Q de la Universidad de Queensland, como un índice individual que cuantifica la investigación y la docencia con la finalidad de permitir comparar académicos, facultades, departamentos, etc. (The University of Queensland, 2014). Si tenemos en cuenta que el puesto de trabajo, la renovación de los contratos o el estatus académico pueden depender del índice Q, podría cumplirse el siguiente aspecto; b) los investigadores pueden llegar a negociar y pactar citas en las revistas para subir el impacto de su trabajo (Lawrence, 2008) o crear grandes grupos de publicación en las que se citan entre ellos (Quindós, 2009). Lo que en un principio fue una forma de buscar una manera objetiva de medir el impacto de los trabajos llegaría a pervertirse por medio de pactos que desvirtúan la idea inicial. Así, el trabajo más citado podría ser el del autor más hábil en posicionar su producto en el mercado de las publicaciones, según la lógica de los impactos y no necesariamente la investigación rigurosa que constituya un mayor aporte para la mejora del campo educativo, sus instituciones y prácticas. Hay que considerar que la meritocracia es una buena forma de crear minorías hegemónicas (Cruz \& Stake, 2012); c) surge la necesidad de publicar en inglés, ya que este idioma concentra el mayor número de revistas en los índices de impacto. Brunner-Ried y Salazar-Muñiz (2012) consideran que esto provoca dificultades en el campo de la Educación, pues muchas investigaciones son de carácter local, regional o nacional y el hecho de escribir sus derivados en inglés no la hacen asequible a esta población local, y d) la devolución de resultados a la sociedad se vuelve compleja cuando la valoración principal es publicar en índices de impacto y no tiene como objetivo ser de utilidad para los actores y el contexto investigado. Kalteimer (2012) plantea como problemática la forma de redactar informes de investigación, puesto que el lenguaje académico suele ser difícil de entender para los participantes de otros ámbitos. Esto desemboca en la creación de una ciencia para científicos y empresas, que excluyen a los participantes.

Nos encontramos inmersos en la dinámica de "publicar o morir", más orientados a este último término, si tenemos en cuenta el trabajo de García-Cepero 
(2008), en el que los artículos divulgados en Web of Science en el área de investigación educativa de países iberoamericanos son minoritarias, con apenas un 3\%. Tener más necesidades de difundir las investigaciones por los investigadores que revistas en español supone que las publicaciones pueden marcar sus normas y ser asumidas en función de las necesidades de publicar. Por momentos cabe cuestionar si las investigaciones priorizan el hecho de basarse en criterios de rigurosidad y calidad (Guba, 1983) o si, más bien, se guían por criterios mercantiles.

- Los zombis universitarios. Ryan (2012) describe la zombificación como una forma pasiva de resistencia y supervivencia de los académicos australianos ante las presiones del sistema universitario. Este fenómeno tiene un carácter mundial mediante la imposición de la cultura de las auditorías (Denzin, 2009; Sparkes, 2013), que provocan una universidad que genera cansancio, ansiedad, estrés e insomnio entre los académicos (Burrows, 2012). Además, hoy día este fenómeno se desarrolla de forma supuestamente anónima, a partir de la aplicación de un programa informático que solo mide estándares cuantificables —como el índice de impacto de los artículos o la recaudación obtenida por los proyectos de investigación-y deja muchos aspectos importantes por fuera de la evaluación (Munson, 2014). En el caso español, en la acreditación a figuras de inicio de la vida académica posdoctoral en educación, los artículos indexados suponen un $30 \%$ de la puntuación, más un $12 \%$ por publicación de libros. Se asume que este es el aspecto clave para acreditarse, ya que esta se consigue con un 55\% de los puntos.

Esta situación se manifiesta en diferentes procesos que se aplican sobre los académicos, como en Australia (Ryan, 2012), por medio de la gobernanza, las auditorías y la vigilancia, las cargas de trabajo y la aquiescencia de los líderes académicos. Una situación similar es la inglesa, con el programa Research Excellence Framework (REF, 2014). En Chile, las universidades tradicionales y sus académicos compiten por lograr mejores posiciones en la clasificación nacional mediante la mejora de la productividad académica, lo que genera una "fragilidad reputacional" (GuzmánValenzuela \& Barnett, 2013a) en el que la pieza clave es la investigación (Guzmán-Valenzuela \& Barnett, 2013b). España nos parece muy interesante, debido a que la aplicación de las evaluaciones es de dos formas: a) para el profesorado funcionario, se evalúan los sexenios de investigación, que les proporcionan el acceso a méritos; si no los consiguen, deben impartir más docencia (España, Ministerio de Ciencia e Innovación, 2008). También se pueden evaluar para la promoción a profesor titular o a catedrático, aunque su contrato es indefinido y sin posibilidad de despido (Aneca, 2014b), y b) para el profesorado no funcionario, en particular los recién doctorados, tiene que enfrentarse a las agencias de evaluación para conseguir una acreditación que valore su currículo y le permita trabajar en la universidad o no hacerlo, lo que supone una cuestión de supervivencia en el sistema (Aneca, 2014a).

\section{La figura del investigador}

La profesión de docente universitario exige cumplir con cinco funciones básicas: docencia, investigación, gestión, administración y extensión universitaria. Dado que para desempeñar la docencia universitaria es habitual que no exista una preparación específica o incentivos que redunden en la motivación de los profesores hacia una práctica innovadora y que las evaluaciones se hacen sobre el impacto de las publicaciones de investigación (ver apartado anterior), consideramos que se comienza a generar un reduccionismo que identifica a profesor universitario con investigador. En este apartado nos enfocaremos en el ámbito de la investigación y sus peligros. Se entiende la investigación como el pilar central para todo aquel que pretenda hacer carrera universitaria y, en gran medida, es el eje en torno al cual va construyendo su identidad profesional, por lo que se convierte en la dimensión más importante de la actuación de muchos profesores. No en vano los investigadores, al abordar sus trabajos, tienen que tomar determinadas decisiones que, debido al peso de la tradición, no suelen plantearse. En las próximas líneas propondremos algunas de estas decisiones.

- Perspectivas de investigación y contexto. Al investigar no solo se define qué se va a investigar, sino también desde qué perspectiva. Vasilachis de Gialdino (2009) considera que el investigador desarrolla su práctica de la forma en que conoce el mundo. La pretendida objetividad del investigador no se puede conseguir, ya que la forma de pensar siempre está presente en la forma de ver e interpretar la realidad, aspecto que ya hemos tratado. Sobre este particular, nos parece acertada la metáfora de García-Carrasco (2007): la persona que estudia las hormigas ve el mundo como un hormiguero.

Cuando es el investigador el que selecciona los objetivos, se encuentran presentes sus creencias, pero en muchas ocasiones se excluye al grupo social que se investiga. A este respecto, la investigación se encuentra condicionada desde el comienzo por la concepción previa que tiene de la realidad investigada que, en parte, procede de su forma de entender el mundo, de sus lecturas de investigaciones previas y 
del conocimiento del campo a investigar. Su forma de entender el mundo y las lecturas de investigaciones marcan el acercamiento al campo desde una perspectiva externa a la del grupo. Es la comunidad científica la que se acerca al campo sin tener en cuenta a los participantes. En algunos casos, como forma de paliar esta situación, algunos investigadores consideran que la teoría puede construirse después del trabajo de campo. Este es el caso de la etnografía - pues si se conocen los escritos sobre la cultura después de la recogida de datos, el investigador se encuentra sin ese sesgo (Goetz \& LeCompte, 1988) - o el de la investigación-acción — que busca la solución de problemas de un grupo concreto (Stringer, 2007)—.

Respecto al conocimiento del campo a investigar, no es lo mismo ser un conocedor teórico que un participante del grupo social. Sobre este asunto, Denzin (2008) considera que es necesario que los investigadores pertenecientes a grupos excluidos tomen voz y presenten sus autoetnografías como forma de ofrecer la perspectiva de los participantes en la comunidad científica. Guba y Lincoln (1989) ven necesaria una participación basada en el diálogo entre investigadores y participantes, en la que surjan puntos de vista diferentes, para compararlos y contrastarlos. Más allá se presenta el trabajo de Horton, Kohl y Kohl (1998), en sus procesos de investigación-acción; proponen que los objetivos del proceso son cosa de los participantes. En todos estos casos, el planteamiento es que, cuando la investigación tiene como objetivo transformar la realidad, la participación ha de estar presente desde el primer momento (Stringer, 2007).

- El investigador como Supermán. Umberto Eco (1965) describe a Supermán de un modo muy similar a como las tradiciones de la investigación asumen al investigador: como un ser sin historia que utiliza sus superpoderes $y$, en general, sin conciencia crítica del mundo en el que vive.

Comenzamos por destacar que Supermán es ahistórico, no tiene pasado ni futuro, solo vive en el presente. Apareció en un meteorito y oculta su origen y su esencia de superhéroe a los demás. Esta situación es habitual en las investigaciones, ya que el investigador entra en el campo sin una perspectiva histórica de él y desaparece cuando ha recogido los datos necesarios. Este suele ser el caso de muchos de los procesos en los que el investigador está vinculado con la investigación el tiempo que dura el estudio, por lo que el conocimiento, aun en profundidad, solo se produce en un momento histórico y con la perspectiva que ofrece ese momento y su visión. Incluso se pretende conocer el pasado desde la perspectiva del presente. Lo anterior tiene que ver con la idea de objetividad de la ciencia, situación que se torna más compleja si nos referimos a las Ciencias Sociales (Guzmán-Valenzuela, 2014).

Supermán no tenía historia, así como tampoco el investigador en el grupo a investigar, pero sí tiene creencias previas. Aunque Taylor y Bogdan (1986) consideren que el investigador cualitativo aparta sus propias creencias, hay otros autores que consideran que esto no es posible y que la investigación cualitativa busca la subjetividad (Flick, 2004; Guba, Lynham, \& Lincoln, 2011). Así, Guba, Lynham y Lincoln (2011) consideran que, en la investigación, la clave es el paradigma del investigador, es decir, cómo construye su conocimiento del mundo, mientras el método con el que obtiene la información apenas condiciona. Esto supone que, cuando nos referimos a un enfoque interpretativo, los datos pasan por el tamiz de la concepción del mundo del investigador, aun cuando haya diálogo con los participantes en el proceso, pues tanto el planteamiento de objetivos como el análisis de datos se realiza bajo su único criterio (o del equipo investigador). Así, en las interpretaciones se hace referencia a cómo entiende el campo el 
investigador y las concepciones y conocimientos previos que tiene. En otra línea, Potter (1996) propone que el lenguaje de la investigación es clave a la hora de criticar o validar lo que se interpreta; esto es, una misma observación puede describirse de forma crítica o reproductora. Aun cuando en ocasiones son procesos inconscientes, el investigador no puede olvidar que la neutralidad no existe y que no tomar partido por una realidad supone apoyar a los grupos hegemónicos de forma explícita.

- Supermán es un personaje de moral externa. Podría parecer paradójico que un superhéroe que tiene como tarea salvar a la humanidad no tenga autonomía moral, pero la realidad es que, si tenemos en cuenta la teoría del desarrollo moral de Kohlberg (1992), Supermán solo hace lo que se espera de él. No muestra autonomía en sus actuaciones ni toma como criterio sus dilemas morales. Por ello, Eco (1965) reconoce que Supermán no abarca proyectos que no hayan sido homologados, de manera que nunca emprendería una revolución social, por muy justa que fuera. Dentro de la investigación, tendríamos desde la Declaración de los Derechos Humanos a códigos éticos de investigación como los de la British Educational Research Association (BERA, 2011) o la American Psychological Association (APA, 2010), que nos convierten en Supermán. Algunos de estos códigos tienen estándares como el anonimato, que impiden desarrollar ciertas formas de investigación cualitativa como la autoetnografía o que cuestionan el hecho de que se revele la identidad de participantes clave cuando se hace un análisis del discurso en el que resulta crucial la posición desde donde se habla o se construye un discurso.

Los códigos éticos vienen determinados por el conjunto de valoraciones personales que se hacen de un determinado hecho; por este motivo, el investigador se plantea si con su trabajo está interviniendo en la realidad, está protegiendo al grupo, es respetuoso con los informantes, etc. De ahí que Angrosino (2012) plantee que la ética de la investigación ha de respetar una dimensión social relacionada con los estándares éticos y la propia valoración personal del investigador en el campo. Al mismo tiempo, no podemos obviar la crítica que plantea Ibáñez-García (1994), referida a que los códigos éticos están desarrollados por investigadores y no por las personas que viven en el contexto que se investiga. Así, la perspectiva del investigador también se encuentra guiada por la forma de pensamiento del colectivo de investigadores que adopta una actitud paternalista ante los participantes, sin dejarles expresarse.

Como alternativa a la imposición ética del grupo de investigadores sobre el de investigados, Denzin y Lincoln (2011) afirman que la ética ha de estar al servicio de los investigados, con principios como el cuidado y la responsabilidad dialógica y comunitaria. Desde esta perspectiva, no se puede considerar que el cuidado y el bien de los investigados puedan ser establecidos por los investigadores sin tener en cuenta a los participantes, lo que hace necesario un diálogo ético entre investigadores y participantes acerca del posicionamiento de la investigación (Carducci, Pasque, Kuntz, \& Contreras-McGavin, 2013). Sobre este aspecto, encontramos experiencias en las que un comité asesor de participantes vela porque sus voces sean tenidas en cuenta (Gómez, Puigvert \& Flecha, 2011; Munté, Serradell \& Sordé, 2011). Este comité está compuesto por representantes de los diferentes colectivos de participantes que serán los usuarios finales de la investigación. El hecho de que los sectores implicados en conseguir resultados finales colaboren de forma dialógica permite delimitar y aclarar intereses y espacios de diálogo y de investigación, a la vez que una construcción colectiva del conocimiento. 


\section{Conclusiones}

Las instituciones universitarias han de entenderse como entidades colectivas en las que es imprescindible que sus diversos agentes (profesores, estudiantes, gestores, etc.) sean capaces de llevar a cabo procesos de enseñanza/aprendizaje de manera interactiva, colaborativa, dinámica y dialógica. Estaremos, así, en condiciones de ofrecer un sistema universitario más eficaz en términos de docencia e investigación. No obstante, como hemos apuntado en este trabajo, que las universidades se dediquen a investigar y difundir ese conocimiento no quiere decir que sean instituciones que posean características de instituciones que aprenden (Greenwood, 2009). Más aún, podría considerarse que producen conocimiento para la sociedad, sin cuestionarse su propia esencia ni plantearse su inmersión en el (neo)liberalismo. Por ese motivo, hemos optado por considerarlas como universidades-becarias. Nosotros los investigadores —en particular los cualitativos, por nuestro compromiso con la justicia social- somos las bases, los encargados de desenmascarar la realidad que nos rodea y actuar sobre ella. Según Bourdieu (2003), un aspecto clave a la hora de entender las relaciones en la realidad o el juego es comprender sus normas para poder actuar. Por eso, entendemos que no solo debemos intervenir en la realidad de nuestras investigaciones, sino que los futuros investigadores también están a nuestro cargo. Se vuelve fundamental acompañar a los investigadores noveles en su aprendizaje del oficio de investigar desde una perspectiva académica y ofrecerles una visión crítica y realista que les permita vivir, narrar y transformar la realidad.

A lo largo del artículo hemos pretendido evidenciar el "lado oscuro" del oficio del investigador. Hemos buscado presiones del sistema que se asumen de forma acrítica y las hemos presentado de modo que se pueda tomar conciencia crítica de ellas. Así, apuntamos cómo los procesos evaluadores a los que ha de someterse el profesional universitario parecen estar diseñados para el ámbito empresarial y, como tal, se han aplicado al contexto de la educación superior. Si bien entendemos que aspirar a la calidad — algo que parecen buscar con ahínco los modelos de evaluación-es una opción muy legítima, creemos que la mejor forma de alcanzarla no necesariamente consiste en regirse por patrones mercantilistas. Más bien, a partir de la labor investigadora y docente, las instituciones universitarias han de construir conocimiento, formar personas, ciudadanos activos y críticos y no tratar de "fidelizar", "conseguir la satisfacción de los clientes" y otros términos que indican un mercadeo de la enseñanza superior.

Relacionado con esto, nos parece que el empleo de estos modelos legitima unas estructuras de control del profesorado y de las instituciones educativas que nos hace preguntarnos si lo que se pretende es calidad o control. Esta situación impone una enorme presión al investigador y académico universitario, sin olvidar a Freire (2007), cuando menciona que el mayor opresor es el que tenemos dentro. Es lo que sucede en la universidad de las auditorías, donde la presión que nos imponemos los investigadores puede ser aún mayor que la que nos impone el sistema.

Se vuelve necesario hallar espacios para desarrollar nuestros intereses y transformar el sistema universitario o de estándares investigadores. Podríamos hacer algunas preguntas para indagar en la cuestión: ¿Van ligadas la productividad investigadora en Educación y la calidad de la enseñanza? ¿Cómo demandar al profesorado universitario una investigación de mayor calidad, al margen de políticas reguladoras en términos de recursos (económicos, humanos, materiales) que las respalden? ¿Puede el profesional universitario realizar bien su labor investigadora sin descuidar las 
demás y viceversa? ¿Qué posibilidades tiene un investigador novel, con méritos mensurables, para hacer carrera universitaria de forma autónoma?

Hemos pujado por despertar la conciencia crítica entre los investigadores, sobre todo de los más cercanos al ámbito cualitativo. Reconocemos como limitación al estudio nuestro posicionamiento claro hacia una perspectiva menos mercantilista de la que impera en la política universitaria actual y somos conscientes de que, en la evaluación de la calidad investigadora, son muchos los puntos controversiales, las posiciones encontradas e, incluso, las perspectivas y las características personales que puedan tener lugar. Lo que aquí hemos pretendido es aportar una serie de reflexiones que posibilite vislumbrar certezas 0 , tal vez, generar dudas acerca de la adecuación y necesidad de alcanzar los mínimos exigidos para la supervivencia universitaria, sin olvidar que la transformación del "lado oscuro" y de todo aquello que consideramos ilícito se ha de realizar desde adentro.

En síntesis, la labor investigadora dentro del ámbito de la educación superior no ha de perder de vista que la universidad debe formar personas que cuenten con las competencias personales y profesionales que les faciliten vivir y desenvolverse en el mundo que a cada uno le toque. Como mundo que es, existe la necesidad de actuar con intención de transformarlo y mejorarlo, en especial en cuanto a las condiciones de las personas. Un reto sin duda complicado, aunque no imposible. iQué la fuerza nos acompañe!

\section{Sobre los autores}

José J. Barba es profesor investigador de la Universidad de Valladolid en el Campus de Segovia. Sus líneas de investigación se enfocan en la aplicación de la investigación cualitativa en Educación, sobre todo en los métodos que implican la participación. Ha desarrollado diferentes trabajos en formación inicial y continua del profesorado.

Gustavo González-Calvo participa en proyectos de innovación docente como el Grupo Acoge (educación, inclusión e igualdad) y el proyecto MultiScopic (desarrollo de comunidades internacionales de práctica en la formación inicial y permanente del profesorado de Educación Primaria). Sus líneas de investigación apuntan al desarrollo de la identidad reflexiva del profesorado, la investigación mediante historias de vida y etnografías escolares.

Raúl A. Barba-Martín ha desarrollado trabajos de investigación en la línea del aprendizaje cooperativo, la educación inclusiva, la investigación-acción y la formación del profesorado.

\section{Referencias}

Agencia Nacional de Evaluación de la Calidad y Acreditación [Aneca]. (2014a). Programa de evaluación del profesorado para la contratación (PEP). Recuperado de http://www.aneca.es/Programas/PEP

Agencia Nacional de Evaluación de la Calidad y Acreditación [Aneca]. (2014b). Programa de acreditación nacional para el acceso a los cuerpos docentes funcionarios (programa Academia). Recuperado de http://www.aneca.es/Programas/ACADEMIA

American Psychological Association [APA]. (2010). Ethical Principles of Psychologists and Code of Conduct. Recuperado de http://www.apa. org/ethics/code/index.aspx

Angrosino, M. (2012). Etnografía y observación participante en investigación cualitativa. Madrid: Ediciones Morata. 
Apple, M. W. (1989). Maestros y textos. Una economía política de las relaciones de clase y de sexo en educación. Barcelona: Paidós.

Barba, J. J. (2013). La investigación cualitativa en Educación en los comienzos del siglo XXI. En Díaz, M. \& Giráldez, A. (eds.). Investigación cualitativa en educación musical. Barcelona: Graó.

Barba, J. J., \& González-Calvo, G. (2013). Perseguir el sueño o caer en lo que existe: La socialización del maestro novel entre el habitus y la investigación-acción. Revista Interuniversitaria de Formación del Profesorado, 78(27,3), 137-144.

Barnett, R. (2001). Los límites de la competencia. El conocimiento, la educación superior y la sociedad. Barcelona: Gedisa.

Barnett, R., \& Parry, G. (2014). Policy Analysis Research in Higher Education: Negotiating Dilemmas. magis, Revista Internacional de Investigación en Educación, 7(14), 69-84.

Bourdieu, P. (2003). Cuestiones de Sociología. Madrid: Istmo.

Bourdieu, P. (2006). La distinción. Criterio y bases sociales del buen gusto. Madrid: Taurus.

British Educational Research Association. (2011). Ethical Guidelines for Educational Research. Recuperado de http://www.bera.ac.uk/wpcontent/uploads/2014/02/BERA-Ethical-Guidelines-2011.pdf

Brunner-Ried, J. J., \& Salazar-Muñiz, F. A. (2012). Investigación educacional en Iberoamérica: entre la invisibilidad y la medición. magis, Revista Internacional de Investigación en Educación, 4(9), 559-575.

Burrows, R. (2012). Living with the H-Index? Metricassemblages in the Contemporary Academy. The Sociological Review, 60(2), 355-372.

Carducci, R., Pasque, P. A., Kuntz, A. M., \& Contreras-McGavin, M. (2013). Disrupting Façades of Clarity in the Teaching and Learning of Qualitative Research. Qualitative Research in Education, 2(1), 1-26.

Chomsky, N. (2007). La (des)educación. Barcelona: Crítica.

Contreras-Domingo, J. (1994). La investigación en la acción: ¿Qué es? Cuadernos de Pedagogía (224), 8-12.

Cruz, F., \& Stake, R. E. (2012).Teaching for Equity, Learning about Discrimination in a Meritocratic Society. Qualitative Research in Education, 1(2), 112-134.

Denzin, N. K. (2008). La política y la ética en la representación pedagógica: hacia una pedagogía de la esperanza. En Kincheloe, J. L., \& McLaren, P. (eds.). Pedagogía crítica. De qué hablamos, dónde estamos. Barcelona: Graó.

Denzin, N. K. (2009). The Elephant in the Living Room: Or Extending the Conversation about the Politics of Evidence. Qualitative Research, 9(2), 139-160.

Denzin, N. K. (2010a). The Qualitative Manifesto: A Call to Arms. Walnut Creek: Left Coast Press.

Denzin, N. K. (2010b). On Elephants and Gold Standards. Qualitative Research, 10(2), 296-272.

Denzin, N. K., \& Lincoln, Y. S. (2011). The Discipline and Practice of Qualitative Research. En Denzin, N. K., \& Lincoln, Y. S. (eds.). The SAGE Handbook of Qualitative Research (4a ed.). Thousand Oaks: SAGE.

Díez-Gutiérrez, E. J. (2011). La mcdonalización de la educación superior. Revista Interuniversitaria de Formación del Profesorado, 72(25,3), $59-76$.

Eco, U. (1965). Apocalípticos e integrados. Barcelona: Lumen.

Elliot, J. (1990). La investigación-acción en educación. Madrid: Ediciones Morata. 
España, Ministerio de Ciencia e Innovación. (2008). Orden CIN/2657/2008, de 18 de septiembre, "Por la que se regula el procedimiento administrativo para la evaluación de la actividad investigadora del personal investigador en formación". Recuperado de https://sede.educacion.gob. es/dctm/ministerio/horizontales/ministerio/organismos/cneai/2009orden-cin-2657-2008-1.pdf?documentld=0901e72b8009156a

Flick, U. (2004). Introducción a la investigación cualitativa. Madrid: Ediciones Morata.

Freire, P. (2007). Pedagogía del oprimido (16a ed.). Madrid: Siglo XXI.

García-Carrasco, J. (2007). Leer en la cara y en el mundo. Barcelona: Herder.

García-Cepero, M. C. (2008). Panorama de las publicaciones seriadas y producción académica en el área de Educación, Iberoamérica. magis, Revista Internacional De Investigación en Educación, 1(2), 13-30.

Goetz, J. P., \& Le Compte, M. D. (1988). Etnografía y diseño cualitativo en la investigación educativa. Madrid: Ediciones Morata.

Gómez, A., Puigvert, L., \& Flecha, R. (2011). Critical Communicative Methodology: Informing Real Social Transformation through Research. Qualitative Inquiry, 17(3), 235-245.

González-Calvo, G., \& Barba, J. J. (2014). La identidad afectivo-personal de un docente y su reflejo en el desarrollo de la identidad profesional. Profesorado. Revista de Currículum y Formación del Profesorado, 18(3). Recuperado de http://www.ugr.es/local/recfpro/ rev181COL12.pdf

Greenwood, D. J. (2009). Are Research Universities Knowledge-Intensive Learning Organizations? En Jemielniak, D., \& Kociatkiewicz, J. (eds.). Handbook of Research on Knowledge-Intensive Organizations. Nueva York: Hershey.

Greenwood, D. J. (2012). Doing and Learning Action Research in the Neoliberal World of Contemporary Higher Education. Action Research, 10(2), 115-132.

Guba, E. G. (1983). Criterios de credibilidad en la investigación naturalista. En Gimeno-Sacristán, J., \& Pérez-Gómez, Á. (eds.). La enseñanza: su teoría y su práctica. Madrid: Akal.

Guba, E. G., \& Lincoln, Y. S. (1989). Fourth Generation Evaluation. Newbury Park: SAGE.

Guba, E. G., Lynham, S. A., \& Lincoln, Y. S. (2011). Paradigmatic Controversies, Contradictions, and Emerging Confluences, Revisited. En Denzin, N. K., \& Lincoln, Y. S. (eds.). The SAGE Handbook of Qualitative Research (4a ed.). Thousand Oaks: SAGE.

Guzmán-Valenzuela, C. (2014). Polos epistemológicos, uso y construcción de teoría en investigación cualitativa en educación. magis, Revista Internacional de Investigación en Educación, 7(14), 15-28.

Guzmán-Valenzuela, C., \& Barnett, R. (2013a). Marketing Time: Evolving Timescapes in Academia. Studies in Higher Education, 38(8), 1120-1134.

Guzmán-Valenzuela, C., \& Barnett, R. (2013b). Academic Fragilities in a Marketized Age: The Case of Chile. British Journal of Educational Studies, 61(2), 1-18.

Hargreaves, A., \& Shirley, D. (2012). La cuarta vía. El futuro prometedor del cambio educativo. Barcelona: Octaedro.

Horton, M., Kohl, J., \& Kohl, H. (1998). The Long Haul: An Autobiography. Columbia: Teachers College Press.

Ibáñez-García, T. (1994). Construccionismo y Psicología. Revista Interamericana de Psicología, 28(1), 105-123. 
Kaltmeier, O. (2012). Hacia la descolonización de las metodologías: reciprocidad, horizontalidad y poder. En Corona-Berkin, S., \& Kaltmeier, O. (eds.). En diálogo metodologías horizontales en Ciencias Sociales. Barcelona: Gedisa.

Kincheloe, J. L. (1991/2012). Teachers as Researches. Qualitative Inquiry as a Path to Empowerment. (4a ed.). Wiltshire: Routledge.

Kohlberg, L. (1992). Psicología del desarrollo moral. Bilbao: Descleé de Brouwer.

Kusher, S. (2009). Prólogo: Recuperar lo personal. En Rivas-Flores, J. I., \& Herrera-Pastor, D. (coords.). Voz y educación. La narrativa como enfoque de interpretación de la realidad. Barcelona: Octaedro.

Latorre, A., Del Rincón, D., \& Arnal, J. (1996). Bases metodológicas de la investigación educativa. Barcelona: Hurtado Ediciones.

Lawrence, P. A. (2008). Lost in Publication: How Measurement Harms Science. Ethics in Science and Environmental Politics, 8(1), 9-11.

McKernan, J. (1999). Investigación-acción y currículum. Métodos y recursos para profesionales reflexivos. Madrid: Ediciones Morata.

Mertens, D. M., Sullivan, M., \& Stace, H. (2011). Disability Communities: Transformative Research for Social Justice. En Denzin, N. K. \& Lincoln, Y. S. (eds.). The SAGE Handbook of Qualitative Research. (4a ed.). Thousand Oaks: SAGE.

Munson, A. (2014). The (Miss) Use of Technology in the National Accreditation System. Qualitative Research in Education, 3(2), 130-152.

Munté, A., Serradell, O., \& Sordé, T. (2011). From Research Policy: Roma Participation trough Communicative Organization. Qualitative Inquiry, 17(3), 256-266.

Potter, J. (1996). Representing Reality. Discourse, Rhetoric and Social Construction. Londres: SAGE.

Quindós, G. (2009). Confundiendo al confuso: reflexiones sobre el factor de impacto, el índice h(irsch), el valor Q y otros cofactores que influyen en la felicidad del investigador. Revista Iberoamericana de Micología, 26(2), 97-102.

REF. (2014). Research Excellence Framework. Recuperado de http://www. ref.ac.uk/

Ryan, S. (2012). Academic Zombies. A Failure of Resistance or a Means of Survival. Australian Universities Review, 54(2), 3-11.

Schön, D. A. (1998). El profesional reflexivo. Cómo piensan los profesionales cuando actúan. Barcelona: Paidós.

Sparkes, A. C. (2013). Qualitative Research in Sport, Exercise and Health in the Era of Neoliberalism, Audit and New Public Management: Understanding the Conditions for the (Im)Possibilities of a New Paradigm Dialogue. Qualitative Research in Sports, Exercise and Health, 5(3), 440-459.

Stenhouse, L. (1987). La investigación como base en la enseñanza. Madrid: Ediciones Morata.

Stringer, E. T. (2007). Action Research in Education. Upper Saddle River: Pearson.

Taylor, S. J., \& Bogdan, R. (1986). Introducción a los métodos cualitativos de investigación. Buenos Aires: Paidós.

The University of Queensland. (2014). Q-Index Information. Recuperado de http://www.uq.edu.au/research/research-at-uq/q-index-information

Vasilachis de Gialdino, I. (2009). Los fundamentos ontológicos y epistemológicos de la investigación cualitativa. Forum: Qualitative Social Research, 10(2). Recuperado de http://nbn-resolving.de/ urn:nbn:de:0114-fqs0902307 
Villasante, T. R., \& Montañés, M. (2000). Algunos cambios de enfoque en Ciencias Sociales. En Villasante, T. R., Montañes, M., \& J. Martí (coords.). La investigación social participativa. Madrid: El Viejo Topo.

Wiesenfeld, E. (2000). Entre la prescripción y la acción: la brecha entre la teoría y la práctica en las investigaciones cualitativas. Forum: Qualitative Social Research, 1(2). Recuperado de http://nbn-resolving.de/ urn:nbn:de:0114-fqs0002303

Zeichner, K. M. (1993). El docente como profesional reflexivo. Cuadernos de Pedagogía, 220, 44-49. 